\title{
Heat stress in dairy cattle - Some potential health risks associated with the nutritional management of the condition
}

Jos J Vermunt

Adjunct Professor (Cattle Health and Production), College of Public Health, Medical and Veterinary Sciences, James Cook University, Townsville, QLD, Australia

*Corresponding author: Jos J Vermunt, 80 Russell Robertson Drive, Havelock North 4130, New Zealand.

Received date: 28 August, 2021 Accepted date: 11 September, 2021| Published date: 16 September, 2021

Citation: Jos J Vermunt (2021) Heat stress in dairy cattle - Some potential health risks associated with the nutritional management of the condition. J Clin Vet Res 1(1). doi https://doi.org/10.54289/JCVR2100102

Copyright: () 2021 Jos J Vermunt. This is an open-access article distributed under the terms of the Creative Commons Attribution License, which permits unrestricted use, distribution, and reproduction in any medium, provided the original author and source are credited.

\begin{abstract}
Heat stress occurs when animals are exposed to environmental temperatures in excess of $25^{\circ} \mathrm{C}$ (the upper critical temperature), particularly in combination with high relative humidity or direct sunshine. Prevention is by providing plenty of good-quality drinking water, providing shade (natural or artificial), and using water sprinklers and/or fans. Changes to the diet, i.e., high energy density and low protein, are also beneficial and often implemented. However, there may be some potential risks associated with the nutritional management of heat stress in dairy cattle; i.e., the animals are at increased risk of developing subacute rumen acidosis, with ensuing laminitis/lameness, and displaced abomasum. This paper discusses how increasing the energy density of the diet (i.e., increasing the grain/forage ratio), as part of the nutritional management of heat stress, may put cows at greater risk of the above-mentioned digestive disorders.
\end{abstract}

Keywords: Heat stress; management; nutrition; subacute rumen acidosis; laminitis; lameness; displaced abomasum Abbreviations: SCC: somatic cell counts, SARA: subacute rumen acidosis, VFAs: volatile fatty acids, NSC: non-structural carbohydrate, NDF: neutral detergent fibre.

\section{Introduction}

Like all other mammals and birds, cattle are 'warm-blooded' homeothermic animals. Despite wide fluctuations in environmental temperature, cattle are normally capable of maintaining a relatively constant body temperature (i.e., between 38.4 and $39.0^{\circ} \mathrm{C}$ ). As environmental temperatures increase, certain thermoregulatory responses, designed to stabilise body temperature, are initiated [1]. However, these thermoregulatory activities may not be sufficient to maintain normal body temperature during periods when ambient air temperature and humidity are particularly high. Hot weather conditions can have significant production effects on cattle, especially in high-yielding dairy cows during summer in wider Australia [2], as well as North America [3]. With global warming, it's likely that many dairy herds in other parts of the world will be likewise affected in the near future.

In dairy cattle, high heat loads lead to depressed feed intake, decreased milk yield, milk fat and protein percentages, and elevated somatic cell counts (SCC) [4]. In severe conditions, immune function and animal welfare can also be compromised, unless the risk is wisely managed.

Cows need ample access to drinking water to allow for increased intakes in order to compensate for increased losses from sweating and increased respiration rates. Also, provision of shade, either natural or artificial, is critical [2]. Cooling by using sprinklers and fans is another option that can be used on dairy farms in hot weather $[2,4]$. 
In dairy herds, it may be possible to modify the ration being fed in order to minimise the anticipated fall in milk production. Increasing the energy density, i.e., the proportion of grain in the ration, will help maintain dry matter intake (DMI) [5], as long as some form of cooling with shade and sprinklers is available. It should be kept in mind, though, that shifting, i.e., increasing the grain/forage ratio may enhance the risk of digestive disorders, such as rumen acidosis and displaced abomasum $[4,6]$. Another option to increase energy intake is to add supplemental fat (a non-starch energy source) in the form of whole cottonseed or tallow to the ration. Finally, over-feeding with protein should be avoided because it requires energy to excrete any excess nitrogen. For more indepth information on heat stress in cattle the reader should refer to comprehensive reviews of the condition $[4,7]$.

\section{So, what are some of the potential health risks} associated with the nutritional management of

\section{heat stress in dairy cattle?}

The most limiting nutrient for lactating cows during periods of heat stress is energy intake [7], and a common approach to increase the energy density is to reduce the forage intake and increase the concentrate content of the ration [5]. The logic is that less fibre (less bulk) will encourage intake, while more concentrates increase the energy density of the diet. Although increasing the energy density of the diet by means of lowfibre, high-fermentable carbohydrate rations (i.e., increasing the grain/forage ratio) may lower the dietary heat load compared to higher fibre diets, this effect must be carefully balanced with the potential risks of subacute rumen acidosis (SARA) and displaced abomasum, conditions that are associated with such high-grain diets $[8,9]$.

\section{SARA and laminitis/lameness}

Rumen acidosis concerns a series of conditions that are manifested by a decrease in rumen $\mathrm{pH}[\mathbf{6}, \mathbf{8}]$. It can present at various levels, from a mild or subacute rumen acidosis to a peracute, life-threatening disease. Rumen acidosis or lactic acidosis can be categorised as two different disease entities, i.e., SARA (also referred to as subclinical acidosis) and acute rumen acidosis (also referred to as clinical acidosis) [6].

SARA is the most common form of rumen acidosis encountered in dairy cows $[6, \mathbf{1 0}]$. It consists of intermittent periods of low rumen $\mathrm{pH}$ that are between acute and chronic in duration. The lowered rumen $\mathrm{pH}$ during SARA selfcorrects within a few hours and affected cows typically exhibit no overt clinical signs [6]. However, chronic health problems, secondary to rumenitis, may appear weeks to months later. The condition is characterised by a rumen $\mathrm{pH}$ between 5.0 and 5.5, where the total concentration of volatile fatty acids (VFAs) has increased, where the ratio between acetate, propionate and butyrate has shifted toward propionate and butyrate, where accumulated concentrations of lactate in the rumen fluid do not exceed 5 to $10 \mathrm{mmol} / \mathrm{l}$, and where the rumen microbial flora is characterised by a dominance of gram-negative bacteria, although the number of gram-positive bacteria is increasing $[\mathbf{6}, \mathbf{8}]$. The lowering of rumen $\mathrm{pH}$ during SARA is primarily due to the accumulation of VFAs, and not to lactate accumulation [8]. However, frequent measurements of rumen lactate during the day under conditions associated with SARA will reveal transient spikes of rumen lactate between about 10 and $40 \mathrm{mmol} / \mathrm{l}$ [8]. The exact role of these lactate spikes is not known, but they probably contribute to the lowered rumen $\mathrm{pH}$.

SARA has also been associated with the occurrence of laminitis-associated claw lesions, such as sole haemorrhages and white line disease, and lameness [11, 12]. The condition plays an important role in the initiation of laminitis and subsequent lameness. Excessive grain or non-structural carbohydrate (NSC) feeding, slug feeding of grain, feeding sources of NSC that are rapidly fermented in the rumen, and feeding finely chopped silage (in particular maize silage) are common factors in the development of laminitis, because of their propensity for inciting SARA.

Over the past 2 to 3 decades there has been a marked increase in the feeding of starch-based concentrates to cows on pasture [10]. Traditionally, in Australia and to a lesser extent New Zealand, the provision of starch-rich concentrates is related to so-called 'slug feeding' practices in dairy herds, i.e., large amounts of grain or concentrates (2 to $8 \mathrm{~kg}$ per head) are offered without sufficient buffering to cows in the bail twice daily at milking time. Unless such herds are well managed (for example by including rumen buffers and/or ionophores to the concentrate part of the diet), there is a real risk of SARA occurring, such that claw lesions associated with subclinical 
laminitis are often seen in these situations [11].

Laminitis has a complex aetiology and uncertain pathogenesis. The pathophysiology of laminitis in cattle has long been assumed to be analogous to that in the horse, namely a disturbance in the micro-circulation of the corium, with ensuing degenerative and possibly inflammatory changes at the dermal-epidermal junction, which may or may not be followed by rotation and 'sinking' of the distal phalanx [11]. Sequelae include impaired horn production with diffuse softening and discolouration, and haemorrhages in the sole and heel (subclinical laminitis); double soles and walls; ulcers in the sole and toe; white line lesions (haemorrhages, separation and abscess); and in chronic cases, deformation of the whole claw [11].

For many years, the commonly accepted hypothesis linking nutrition with the aetiology and pathogenesis of subclinical laminitis has been that toxic substances, such as histamine, lactic acid, serotonin and endotoxin, are formed or released in the digestive tract (mainly as a consequence of rumen acidosis [12]. These toxic, vasoactive components, together with a coagulopathy, were believed to severely disturb the microvasculature and haemodynamics of the corium, leading to tissue hypoxia and nutrient starvation, followed by ischaemic necrosis and degeneration of the horn-producing structures. However, almost 40 years after the invention of the concept of subclinical laminitis and its linkage to a nutritional insult, this process has largely remained an unproven hypothesis [11].

Nevertheless, while the aetiology and pathogenesis of laminitis may not be as yet fully understood, an increased incidence of laminitis-associated lesions is commonly observed with increasing levels of concentrate feeding, particularly if no attempt is made to buffer the ration adequately in order to minimise the effects of SARA. This condition is a relatively common condition in dairy cows as they first enter the milking herd, and many cases of subclinical laminitis are caused by relatively mild episodes of SARA [6].

The signs displayed by animals with SARA are usually mild, namely reduction in milk yield, reduced butterfat percentage, decreased rumen contractions, mild diarrhoea, and some degree of lethargy [6]. However, the associated changes in the animals' rumen environment are significant enough to cause subclinical laminitis. No obvious changes are seen in the claws at the time of the insult, but lesions associated with subclinical laminitis (sole haemorrhages and yellowing and softening of the horn) may be detected some 6 to 8 weeks later [11].

Until recently, laminitis was rather uncommon in pasturebased dairy cattle in Australasia. However, the incidence of laminitis (and to be more specific subclinical laminitis) is increasing, probably due to the intensification of the diet, involving the feeding of increased amounts of concentrates.

The role of nutrition and feeding management in the development of laminitis/lameness in the Australasian dairy scene is not well understood. However, it is likely that nutritional factors moderate the incidence and severity of lameness by contributing to the occurrence of subclinical laminitis [11]. Therefore, there may be situations where the role of nutrition should be considered as a potential moderator of the extent and severity of lameness. The risk of nutrition contributing to laminitis/lameness will increase according to the amount of concentrate being fed, particularly when these concentrates are being 'slug-fed' in the bail while cows are being milked. In this situation, laminitis/lameness is associated with SARA and, therefore, any recommendations made to reduce the incidence of subclinical laminitis should be aimed at preventing SARA [6].

The risk of SARA developing is less when the concentrate/forage ratio of the diet is kept well under 60:40 [13], which is usually the case in pasture-based dairying systems. However, such a concentrate/forage ratio does not readily fit into the nutritional management of heat-stressed dairy cows, which involves increasing the energy density (i.e., the proportion of grain) of the diet, while feeding high-quality forages, i.e., those with a low percentage of neutral detergent fibre (NDF).

Dairy cows require a minimum amount of effective fibre and forage in their diet for proper chewing and rumination activity, proper rumen function and to maintain a rumen $\mathrm{pH}$ $>6.2$. They need to chew (masticate and ruminate) for 10 to 12 hours/day in order to maintain normal rumen function [6]. The effective fibre of a feed is directly related to the chewing time and, therefore, saliva production associated with that 
particular feed. High-fibre diets, such as hay and coarsely chopped silage, stimulate rumination, which in turn increases saliva flow. Saliva is rich in bicarbonate, which acts as a buffer by neutralising the acid produced in the rumen. Fine chopping of forages reduces their effective fibre content. Adding buffers to rations containing finely chopped silage may help if saliva production is low.

Under US feeding systems, it is recommended that the diet contains a minimum of $25 \%$ neutral detergent fibre (NDF) [8]. This recommendation, however, may be inadequate for diets in which pasture is the predominant forage. One reason that rumen $\mathrm{pH}$ may be low in cows on high-quality pasture is that the NDF of pasture is less effective than that in silage and hay. In this situation, adding a small amount of straw to the diet will be beneficial.

Finally, heat stress has been associated with an increased incidence of lameness in North America [14]. Potentially, heat stress could also be a predisposing factor to lameness in Australasia. Heat stress alters the animals' breathing rate (it may double), heart rate, immune response (it is reduced), and behaviour (e.g., cows are standing for longer periods of time, thereby promoting the pooling of blood in the digits). Heatstressed cows also lose significant amounts of saliva from open-mouth breathing and drooling.

Reduced feed intake, a preference for concentrates rather than forage, a loss of salivary buffering from increased respiratory rates and drooling, and a reduction in the total buffering pool all contribute to a greater potential of SARA occurring during periods of hot and humid weather. Considering the above, it can be argued that increasing the energy density (i.e., the grain/forage ratio) of the diet, as part of the nutritional management of heat stress, may further increase the risk of SARA in heat-stressed dairy cows.

\section{Displaced abomasum}

A number of managerial, environmental, physiological and, possibly, hereditary risk factors are thought to play a role in the development of displaced abomasum, particularly in dairy cattle $[9,15]$. As pregnancy progresses, the growing uterus pushes the rumen cranially and dorsally, and the abomasum may assume a position more to the left than normal. The uterus begins to slide under the caudal aspect of the rumen, thereby reducing rumen volume by about one-third at the end of gestation. The decrease in feed intake that occurs normally during the peri-parturient period may reduce rumen volume even further, and allowing the abomasum to move to the left, between the rumen and the abdominal wall $[9,15]$. The pylorus, however, continues to extend across the abdomen to the right side of the cow.

Three factors are believed to be responsible for allowing the abomasum to shift further to the left side of the abdominal cavity [9]. First, the rumen must fail to take up the void left by the

involuting uterus following parturition, allowing the abomasum to fill that void, thus moving even further to the left. If the rumen moved into its normal position on the left ventral floor of the abdomen, the abomasum would not be able to slide under it. Second, the omentum attached to the abomasum must have been stretched to permit movement of the abomasum to the left side. Both these two factors provide opportunity for displacement. A third factor necessary to cause displacement of the abomasum is abomasal atony.

Normally, gases produced in the abomasum (from fermentation of feedstuffs) are expelled back into the rumen as a result of abomasal contractions. It is believed that these contractions are impaired in cows developing displacement of the abomasum. Trapped gas then causes the drift upwards, either along the left or the right abdominal wall. In most cases, the cause of abomasal atony is less clear. Potential causes include increased volatile fatty acids (VFAs) [16], and decreased smooth muscle tone associated with hypocalcaemia [9].

Nutrition undoubtedly plays an important role in the aetiology of displaced abomasum $[\mathbf{1 7}, \mathbf{1 8}]$. The practise of feeding concentrates and low-fibre diets are commonly incriminated, probably through an increase in the concentration of VFAs in the abomasum, which is known to inhibit abomasal motility $[16,17]$. As a consequence, the flow of digesta from the abomasum to the duodenum is reduced and ingesta start to accumulate in the abomasum. An observation from many cattle practitioners in Australasia is that as the level of concentrate feeding in a region increases, so does the incidence of left displacement of the abomasum.

Decreased abomasal motility, or atony, may result in ingesta accumulating in the abomasum. A high-concentrate, reduced 
-forage diet can also promote the appearance of VFAs in the abomasum by reducing the depth of the rumen raft, or fibre mat, which is made up primarily from the long-fibre material of forages [9]. Normally, the rumen raft captures any grain particles, which are then fermented at the top of the rumen liquor. In cows with an inadequate rumen raft, some grain particles may pass on to the abomasum, where they will then be fermented to some extent. The large volumes of methane and carbon dioxide produced in the abomasum following grain feeding may then become trapped in the organ, potentially causing distension and displacement [9].

The physical form of the ration must also be considered. A good rumen raft is required to stimulate rumen contractions in the cow. Abomasal contractions are closely linked to rumen contractions through the vagus nerve reflex [9]. If the rumen has an inadequate raft to stimulate contractions, then the abomasum will not contract properly either. A thick rumen raft is generally present when cows are fed a high-roughage diet; however, the depth of this raft is rapidly reduced as the amount of concentrates in the ration increases [17]. Feeding high-carbohydrate rations, which contain inadequate levels of roughage (crude fibre levels below 17\%), is an important risk factor for displaced abomasum [17]. Fibre useful to the cow, in terms of rumen health, must stimulate chewing and saliva production in the cow. Not meeting the chewing requirements of the cow is a major problem contributing to abomasal displacement. Feeding a diet containing adequate amounts of fibre of adequate length is one of the most important methods recommended to decrease the incidence of displaced abomasum [9]. However, by and large, such a feeding regimen is not consistent with that recommended for heatstressed dairy cows. Under those conditions, it is commonly advised to increase the energy density (grain/forage ratio) of the overall diet.

In conclusion, the nutritional management of lactating dairy cows may be particularly challenging during periods of intense heat. The main objective should be to maintain good levels of production as well as homeostasis, without adding to the animals' internal heat load, and producing a tendency toward SARA, with subsequent subclinical laminitis or lameness, and/or displaced abomasum.

\section{References}

1. Shearer JK, Beede DK (1990) Thermoregulation and physiological responses in dairy cattle in hot weather. Agri-Pract 11(4): 5-17.

2. Little S, Campbell J. (2008) Cool cows: Dealing with heat stress in Australian Dairy Herds. Dairy Australia.

3. West JW (2003) Effects of heat-stress on production in dairy cattle. J Dairy Sci 86(6): 2131-2144.

4. Vermunt JJ, Malmo J, Parkinson TJ (2019). Heat stress. In: Parkinson TJ, Vermunt JJ, Malmo J, Laven R (eds) Diseases of Cattle in Australasia - A Comprehensive Textbook, $2^{\text {nd }}$ Edn. Pp 1111-1116. Massey University Press, Auckland, New Zealand.

5. West JW (1998) Nutritional strategies for managing the heat-stressed dairy cow. J Dairy Sci 77(2): 21-35.

6. Reference Advisory Group on Fermentative Acidosis of Ruminants (RAGFAR) (2007). Ruminal acidosis - aetiopathogenesis, prevention and treatment. A review for veterinarians and nutritional professionals. Aust Vet Ass (ed). Blackwell Publishing Asia Pty. Ltd., Carlton, Vic, Australia.

7. Kadzere CT, Murphy MR, Silanikove N, Maltz E (2002). Heat stress in lactating dairy cows: a review. Livest Prod Sci 77(1): 59-91.

8. Malmo J, Vermunt JJ, Parkinson TJ (2019). Rumen acidosis. In: Parkinson TJ, Vermunt JJ, Malmo J, Laven R (eds) Diseases of Cattle in Australasia - A Comprehensive Textbook, $2^{\text {nd }}$ Edn. Pp 117-127. Massey University Press, Auckland, New Zealand.

9. Malmo J, Vermunt JJ, Parkinson TJ (2019). Displaced abomasum. In: Parkinson TJ, Vermunt JJ, Malmo J, Laven R (eds) Diseases of Cattle in Australasia - A Comprehensive Textbook, $2^{\text {nd }}$ Edn. Pp 142-144. Massey University Press, Auckland, New Zealand

10. Bramley E, Lean IJ, Fulkerson WJ, Stevenson MA, Rabiee AR, et al. (2008). The definition of acidosis in dairy herds predominantly fed on pasture and concentrates. J Dairy Sci 91(1): 308-321. 
11. Laven RA, Vermunt JJ, Malmo J (2019). Laminitis. In: Parkinson TJ, Vermunt JJ, Malmo J, Laven R (eds) Diseases of Cattle in Australasia - A Comprehensive Textbook, $2^{\text {nd }}$ Edn. Pp 948-951. Massey University Press, Auckland, New Zealand.

12. Lean IJ, Westwood CT, Golder HM, Vermunt JJ (2013). Impact of nutrition on lameness and claw health in cattle. Livest Sci 156(1): 71-87.

13. Oetzel G (2017). Diagnosis and management of subacute ruminal acidosis in dairy herds. Vet Clin North Am Food Anim Pract 33(3): 451-61.

14. Cook NB, Mentink RL, Bennett TB, Burgi K (2007). The effect of heat stress and lameness on time budgets of lactating dairy cows. J Dairy Sci 90(4): 1674-1682.
15. Rohrbach BW, Cannedy AL, Freeman K, Slenning BD (1999). Risk factors for abomasal displacement in dairy cows. J Am Vet Med Assoc 214(11): 16601663.

16. Scott PR, Penny CD, Macrae AI (2011). Cattle Medicine. Pp 80-81. Manson Publishing Ltd, London, UK.

17. Shaver RD (1997). Nutritional risk factors in the aetiology of left displaced abomasum in dairy cows: A review. J Dairy Sci 80(10): 2449-2453.

18. Barrett DC (2003). Prevention of displaced abomasum: is it just getting the nutrition right? Cattle Pract 11: 127-134. 